\title{
Understanding Structural and Electronic Properties of Dithienyl \\ Benzothiadiazole and Its Complex with C70
}

Ram S. Bhatta ${ }^{*}$ and Mesfin Tsige*

Department of Polymer Science, The University of Akron, Ohio 44325, United States

*Corresponding authors: rsb20@uakron.edu; mtsige@uakron.edu 


\begin{abstract}
Understanding the bulk heterojunction (BHJ) morphology of dithienyl benzothiadiazole (DTBT)-based conjugated polymers, the most widely used thirdgeneration electron-donors in BHJ-based Organic photovoltaic (OPV) devices, is the current focus of the OPV community. However, there are still debates [J. Am. Chem. Soc. 2013, 135, 1806-1815 and J. Am. Chem. Soc. 2012, 134, 3498-3507] on the most stable conformation of DTBT and is the main focus of the present study. Herein, we report the conformational and electronic properties of DTBT performing detailed first-principle calculations at the molecular level. We found that the energy difference between the two debated DTBT conformations is about $1.3 \mathrm{kT}$, regardless of methyl or hexyl substituted thiophenes. This energy difference is mainly due to the extent of intramolecular hydrogen bonding. We further report that the low-energy DTBT conformation has a low energy gap, low equilibrium separation $(\sim 3.2 \AA)$ with $\mathrm{C} 70$ and proper orbital energy offset, thereby suggesting DTBT-based polymers to be efficient electron donors for OPV devices.
\end{abstract}

Keywords: Potential energy surface; Energy gap; Donor-acceptor complex 


\section{INTRODUCTION}

Organic photovoltaic (OPV) devices based on the bulk heterojunction (BHJ) concept are promising candidates to meet the world's growing demand for clean, renewable and sustainable energy since they are expected to significantly reduce the cost associated with the electricity generation [1-3]. In BHJ-based OPV devices, organic conjugated polymers acting as electron-donors are blended with fullerene derivatives (electron-acceptors) [4]. Poly(p-phenylene vinylene) and its derivatives are the first generation electron-donors, whereas polythiophene and its derivatives are the second generation electron-donors, poly(3-hexylthiophene) (P3HT) being the most-studied electron-donor for the past decade [5]. Benzothiadiazole-based conjugated polymers are among the third-generation electron-donors used in BHJ-based OPV devices [6]. Understanding the BHJ morphology of OPV devices is crucial to improve the performance of OPV devices [7]. From the fundamental standpoint, this requires the investigation of the probable polymer backbone conformations because the accessible backbone conformations affect both intramolecular and intermolecular interactions [8]. Recently, experimental studies on the probable backbone conformations of benzothiadiazole-based polymers are reported $[9,10]$. However, these studies presented conflicting results on the most stable conformation of dithienyl benzothiadiazole (DTBT) unit (Figure 1). Stuart et al. [9] using single crystal X-ray diffraction have reported the cis-trans conformation (Figure 1(b)) as the most stable conformation, whereas Osaka et al. [10] also using similar experimental techniques have reported the trans conformation (Figure 1(c)) as the most stable one. In order to resolve this issue, we have performed first-principles calculations based on density functional theory (DFT) [11]. 

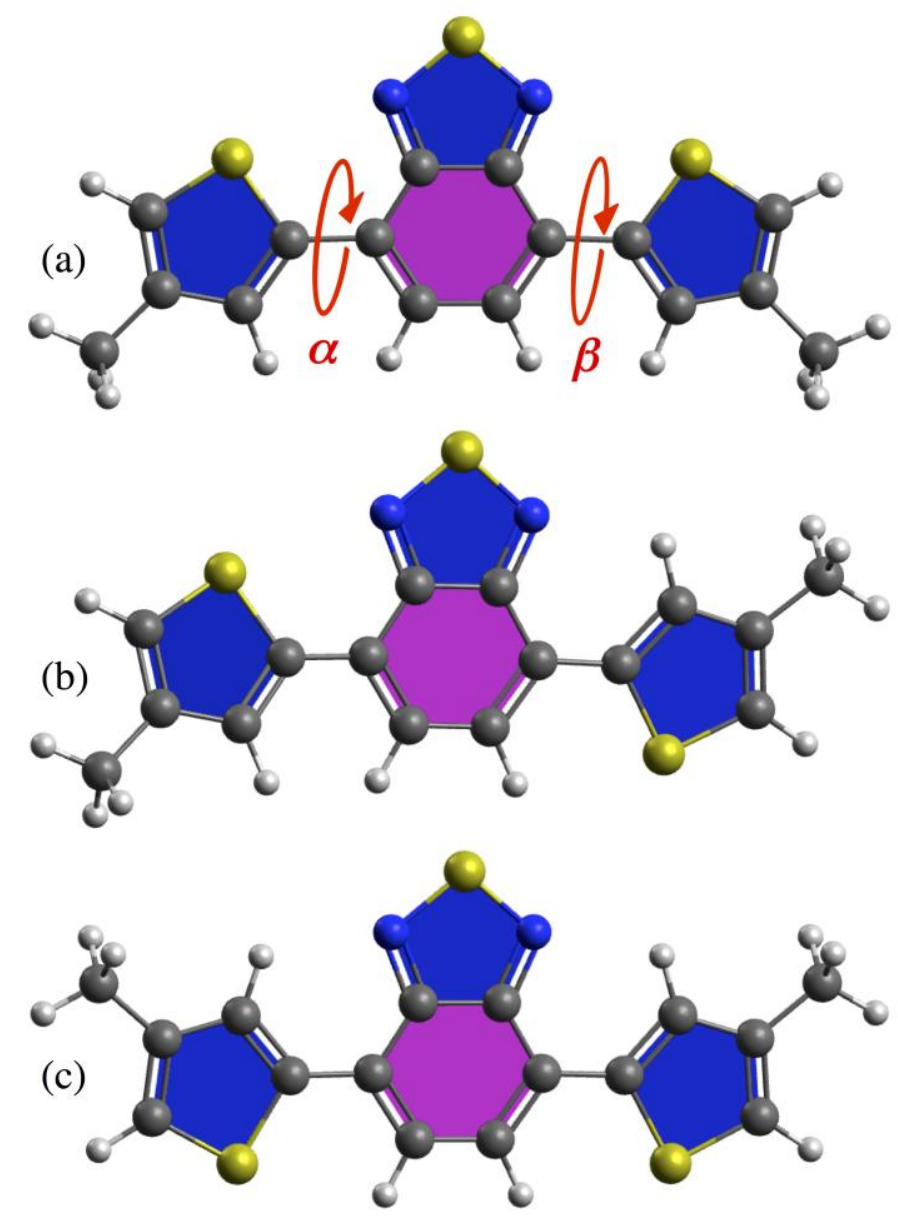

Figure 1. Conformations of DTBT molecule at (a) $\alpha=\beta=0^{\circ}$, (b) $\alpha=0^{\circ} ; \beta=180^{\circ}$ and (c) $\alpha=\beta=180^{\circ}$. By symmetry, conformation at $\alpha=180^{\circ}$ and $\beta=0^{\circ}$ is equivalent to (b).

In this work, we perform first-principles calculations to investigate the probable DTBT conformations at the molecular level. We compute the two-dimensional potential energy surface as functions of inter-ring torsional coordinates to identify the most probable conformations. We further investigate the equilibrium separation of DTBT and C70 in DTBT/C70 complexes to understand the influence of conformations on electronic properties. We discuss implications of probable conformations in orbital energies, ground-to-excited state dipole differences and the energy level offset in DTBT/C70 complexes. 


\section{COMPUTATIONAL METHODS}

All first-principles calculations were performed using DFT combined with Becke's three-parameter Lee-Yang-Parr exchange correlations functional (B3LYP) [12] with dispersion correction using Grimme approach [13] as implemented in the Gaussian 09 program package [14]. The 6-31G(d) basis set was used with diffuse functions on all heavy atoms. Some benchmark computations of conformational energies were also performed using higher electron correlated methods [15] and range-separated functionals (CAM-B3LYP, LC-BLYP, LC-wPBE) [16-18] for the sake of comparison. These results yielded the lowest-energy trans-conformation of DTBT consistent with the dispersion corrected B3LYP results. The dispersion-corrected B3LYP functional was found to yield accurate conformational and electronic properties, as also found in our previous studies [19-21] on similar conjugated polymers. The B3LYP has also been validated as a reliable approach to produce structural and electronic properties in similar conjugated systems [22-25]. The excited state calculations were performed using time dependent DFT (TDDFT) [26].

Geometry optimizations were performed at constrained values of inter-ring toraional angles, $\alpha$ and $\beta$ in the range of $0^{\circ} \leq \alpha \geq 180^{\circ}$ and $0^{\circ} \leq \beta \geq 180^{\circ}$. The twodimensional potential energy surface was obtained by fitting 169 computed grid points (the root-mean-squared residual $\sim 0.6 \mathrm{meV}$ ) using the following equation $[19,27]$ :

$$
V=V_{0}+\sum_{n=1}^{7} k_{n}^{\alpha} \cos n \alpha+\sum_{n=1}^{7} k_{n}^{\beta} \cos n \beta+\sum_{n=1}^{2} k_{n}^{\alpha \beta} \cos n \alpha \cos n \beta
$$




\section{RESULTS AND DISCUSSION}

\subsection{Two-Dimensional Potential Energy Surface}

The two-dimensional potential energy surface shown in Figure 2 has a maximum at $\alpha=\beta=90^{\circ}$ and a minimum at $\alpha=\beta=180^{\circ}$. The surface has other three local minima at $\alpha=\beta=0^{\circ}, \alpha=0^{\circ}$ and $\beta=180^{\circ}$, and $\alpha=180^{\circ}$ and $\beta=0^{\circ}$. The potential energies at $\alpha=$ $0^{\circ}$ and $\beta=180^{\circ}$, and $\alpha=180^{\circ}$ and $\beta=0^{\circ}$ are equal by symmetry of the molecule. The minima are connected via local maxima at $\alpha=0^{\circ}$ and $\beta=90^{\circ}, \alpha=90^{\circ}$ and $\beta=0^{\circ}, \alpha=$ $180^{\circ}$ and $\beta=90^{\circ}, \alpha=90^{\circ}$ and $\beta=180^{\circ}$. The energies at $\alpha=\beta=0^{\circ}$ and $\alpha=0^{\circ}$ and $\beta$ $=180^{\circ}$ are higher by $\sim 67 \mathrm{meV}$ and $\sim 32 \mathrm{meV}$, respectively compared to the lowest energy at $\alpha=\beta=180^{\circ}$. In other words, the energies of the DTBT conformations at $\alpha=\beta=0^{\circ}$ and $\alpha=0^{\circ}$ and $\beta=180^{\circ}$ at room temperature are $\sim 2.6 \mathrm{kT}$ and $\sim 1.3 \mathrm{kT}$ higher than the energy at $\alpha=\beta=180^{\circ}$, respectively (Table 1). Risko et al. [28] have also found that the energy at $\alpha=\beta=0^{\circ}$ is higher than the energy at $\alpha=\beta=180^{\circ}$. The DTBT conformation at $\alpha=\beta=180^{\circ}$ has the lowest energy, regardless of methyl or hexyl-substituted thiophenes. The present result shows that the DTBT conformation at $\alpha=\beta=180^{\circ}$ has the lowest energy and the most favorable conformation in agreement with the earlier reported single crystal X-ray results [10]. 


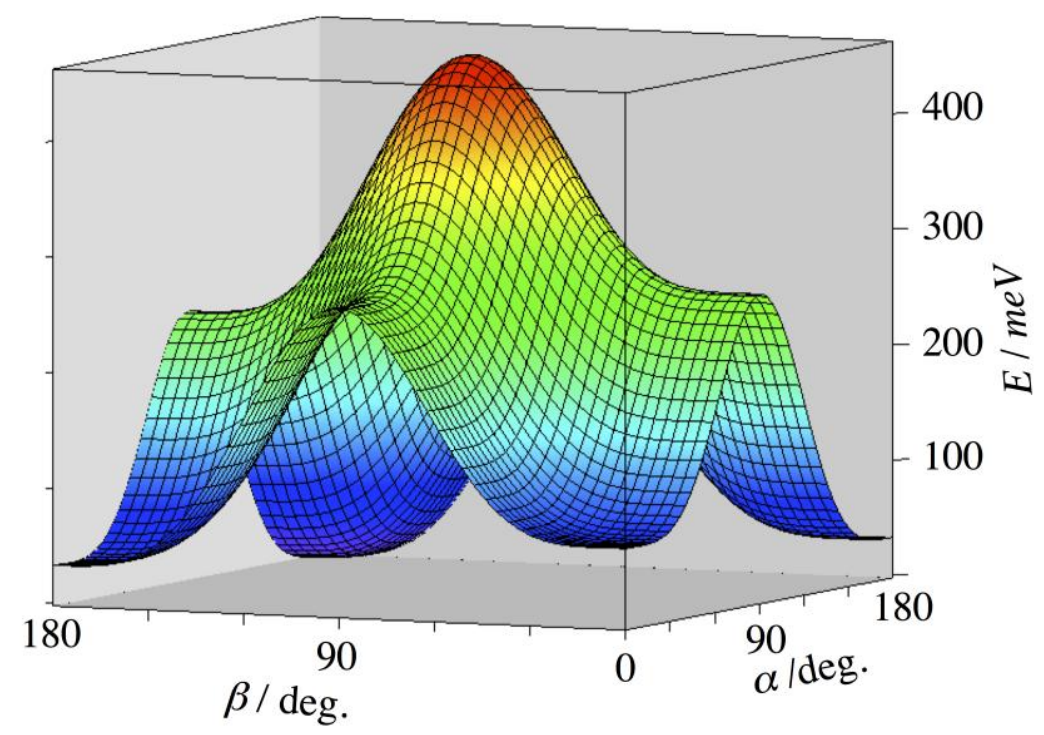

Figure 2. Two-dimensional potential energy surface of DTBT molecule as functions of $\alpha$ and $\beta$.

Table 1. Computed relative energies, HOMO-LUMO gaps and dipole moments of the DTBT conformations

\begin{tabular}{cccc}
\hline Properties & $\begin{array}{c}\alpha=0^{\circ} ; \\
\beta=0^{\circ}\end{array}$ & $\begin{array}{c}\alpha=0^{\circ} ; \\
\beta=180^{\circ}\end{array}$ & $\begin{array}{c}\alpha=180^{\circ} ; \\
\beta=180^{\circ}\end{array}$ \\
\hline Relative $E(\mathrm{meV})$ & 66.8 & 32.0 & 0.0 \\
HOMO-LUMO gap (eV) & 2.70 & 3.67 & 2.65 \\
$\mu_{\mathrm{g}}(\mathrm{D})$ & 2.84 & 1.19 & 0.47 \\
$\mu_{\mathrm{e}}(\mathrm{D})$ & 6.37 & 4.75 & 3.13 \\
$\Delta \mu_{\mathrm{ge}}(\mathrm{D})$ & 3.53 & 3.56 & 3.60 \\
\hline
\end{tabular}

The relative energies of DTBT conformations can be understood in terms of interactions such as pi-conjugation and hydrogen bonding. The DTBT conformations at $\alpha$ $=\beta=0^{\circ}, \alpha=0^{\circ}$ and $\beta=180^{\circ}$, and $\alpha=\beta=180^{\circ}$ are all stabilized by the maximum effective pi-conjugation. The differences in energy among these conformations are attributed to the $\mathrm{N}-\mathrm{H}$ hydrogen bonding. The conformation at $\alpha=\beta=180^{\circ}$ is stabilized by two hydrogen bonding interactions per DTBT molecule. The conformation at $\alpha=0^{\circ}$ 
and $\beta=180^{\circ}$ involves only one hydrogen bond, whereas the conformation at $\alpha=\beta=0^{\circ}$ does not involve any hydrogen bonding. Similarly, the maximum energy conformation at $\alpha=\beta=90^{\circ}$ is the result of the breakage of pi-conjugation as well as the absence of hydrogen bonding interactions.

The hydrogen bond formation can be understood from the atomic charge analysis and the interatomic distance. The nitrogen atoms of DTBT conformations have Mulliken charge of -0.58 au. The nearest hydrogen atom from nitrogen atom in DTBT conformations at $\alpha=\beta=180^{\circ}$ has atomic charge of +0.17 au. The $\mathrm{N}-\mathrm{H}$ separation is 2.3 $\AA$, which is smaller than the sum of van der Waals radii of $\mathrm{N}$ and $\mathrm{H}$ atoms $(2.75 \AA)$. The atomic charge on $\mathrm{H}$ atom reduces by 0.05 au in the case of DTBT conformation at $\alpha=\beta$ $=0^{\circ}$.

\subsection{HOMO-LUMO Gap}

The DTBT conformations not only have different potential energies (Figure 2), but also different HOMO-LUMO gaps. The usual approach for computing the dependence of HOMO-LUMO gap on the molecular conformation is to calculate the orbital energies as functions of $\alpha$ and $\beta$ [19]. The two-dimensional surface of the energy gap between highest occupied molecular orbital (HOMO) and lowest unoccupied molecular orbital (LUMO) was computed from ground state calculations. Figure 3 shows the two-dimensional HOMO-LUMO gap surface as functions of $\alpha$ and $\beta$. As depicted in Figure 3, the HOMO-LUMO gap of DTBT unit is minimum at $\alpha=\beta=180^{\circ}$. The HOMO-LUMO gaps at $\alpha=\beta=0^{\circ}, \alpha=0^{\circ}$ and $\beta=180^{\circ}$ are higher by $\sim 50 \mathrm{meV}$ and $\sim 20$ meV, respectively compared to the gap at $\alpha=\beta=180^{\circ}$ (Table 1). The HOMO-LUMO 
gap increases for the non-planar conformation and reaches to the maximum when $\alpha=\beta=$ $90^{\circ}$. The maximum is connected with four local maxima, three local minima and a global minimum.

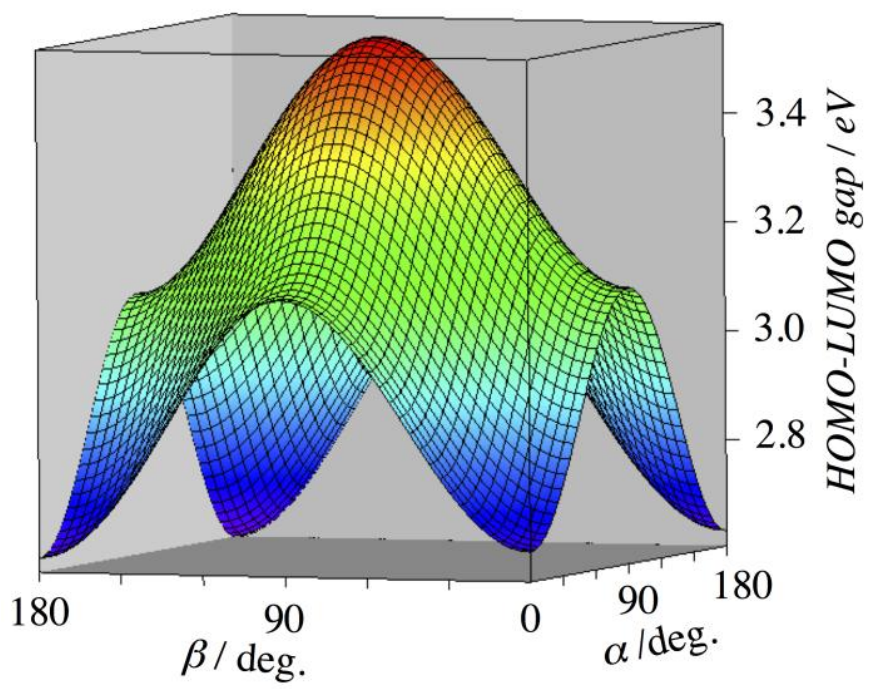

Figure 3. HOMO-LUMO gap surface for DTBT molecule as functions of $\alpha$ and $\beta$ computed using B3LYP.

The form of the HOMO-LUMO gap surface (Figure 3) can be understood in terms of the effect of pi-conjugation and hydrogen bonding on orbital energies. All planar conformations at $\alpha=\beta=0^{\circ}, \alpha=0^{\circ}$ and $\beta=180^{\circ}$, and $\alpha=\beta=180^{\circ}$ favor extended conjugation as a result of the effective overlap of p-orbitals. The lower HOMO-LUMO gap of DTBT at $\alpha=\beta=180^{\circ}$ compared to conformations at $\alpha=0^{\circ}$ and $\beta=180^{\circ}$, and $\alpha=$ $\beta=0^{\circ}$ is accounted for the further decrease in the HOMO and LUMO energy separation due to the hydrogen bonding interactions. At the maximum $\left(\alpha=\beta=90^{\circ}\right), \mathrm{HOMO}$ and LUMO are more separated as a result of the breakage of extended conjugation across the conjugated backbone. 


\subsection{MEP Surface and Dipole Moment}

Further information on the charge distribution across the conformations can be obtained from the molecular electrostatic potential (MEP) surfaces shown in Figure 4. In MEP surfaces, the blue color represents a positive charge (low electron density) whereas the red represents a negative charge (high electron density). The change in the electron density causes fluctuation in the dipole moment value in these conformations. In the ground state, the dipole moment of DTBT molecule is $2.84 \mathrm{D}, 1.19 \mathrm{D}$ and $0.47 \mathrm{D}$ at $\alpha=$ $\beta=0^{\circ}, \alpha=0^{\circ}$ and $\beta=180^{\circ}$ conformation, and $\alpha=\beta=180^{\circ}$, respectively (Table 1 ). The corresponding dipole moments of these conformations are $6.37 \mathrm{D}, 4.75 \mathrm{D}$ and $3.13 \mathrm{D}$, respectively in the excited state. These excited state TD-DFT results are obtained from vertical transitions computed at the B3LYP level starting from the ground state optimized

conformations. The ground-to-excited state change in the dipole moment $\left(\Delta \mu_{\mathrm{ge}}\right)$, an important parameter for charge separation, is calculated from the ground state dipole moment $\left(\mu_{\mathrm{g}}\right)$ and the excited state dipole moment $\left(\mu_{\mathrm{e}}\right)$ as [29],

$$
\Delta \mu_{g e}=\left[\left(\mu_{g x}-\mu_{e x}\right)^{2}+\left(\mu_{g y}-\mu_{e y}\right)^{2}+\left(\mu_{g z}-\mu_{e z}\right)^{2}\right]^{1 / 2}
$$




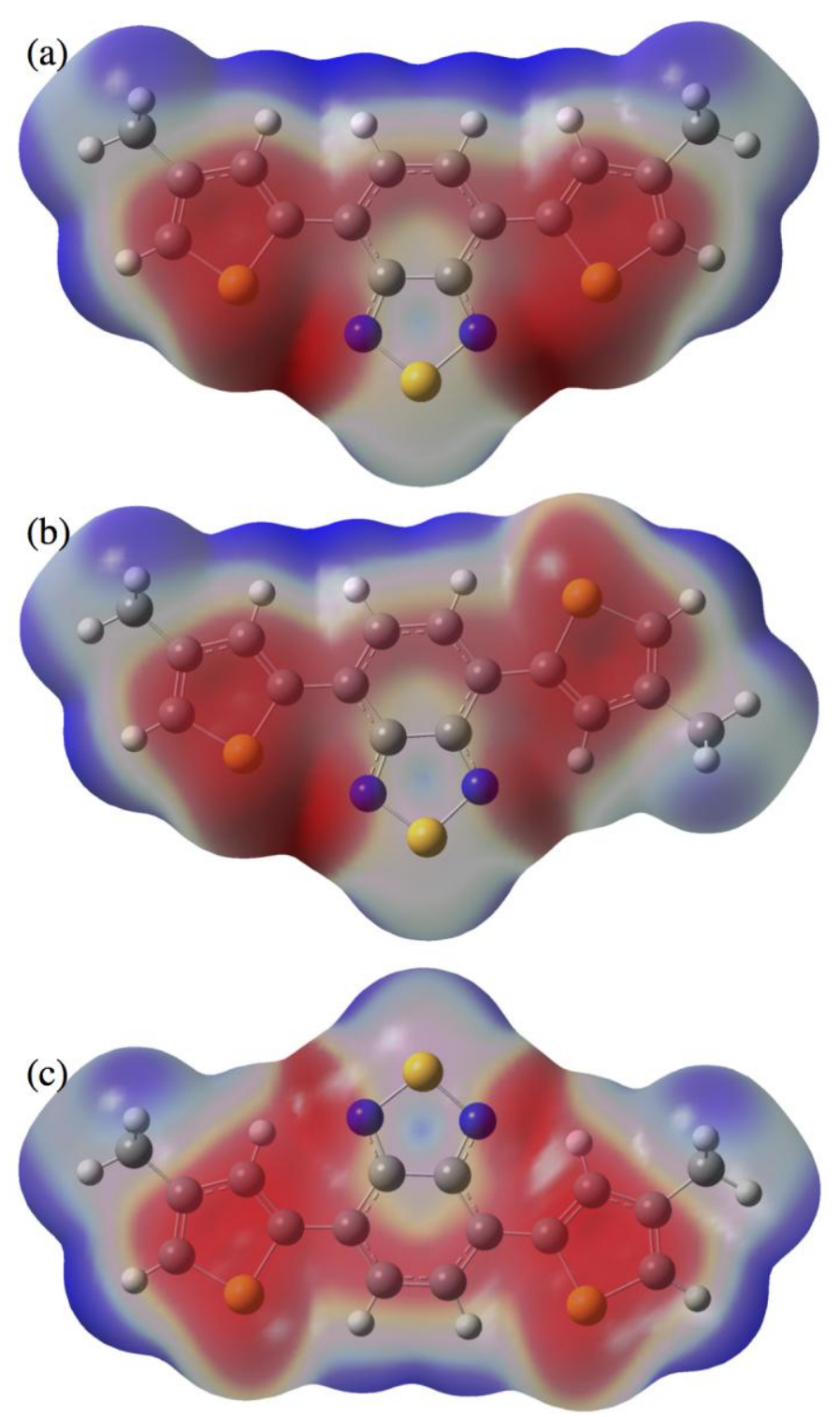

Figure 4. MEP surfaces at (a) $\alpha=\beta=0^{\circ}$, (b) $\alpha=0^{\circ} ; \beta=180^{\circ}$ and (c) $\alpha=\beta=180^{\circ}$.

In Eq. 2, subscripts "x", "y" and "z" represent axial components of the ground and the excited state dipole moments. The computed values of $\Delta \mu_{\mathrm{ge}}$ for DTBT molecule are 3.53 $\mathrm{D}, 3.56 \mathrm{D}$ and 3.60 $\mathrm{D} \alpha=\beta=0^{\circ}, \alpha=0^{\circ}$ and $\beta=180^{\circ}$, and $\alpha=\beta=180^{\circ}$, respectively. This shows that the lowest-energy conformation may promote slightly higher charge separation. This is because the weak dipole moment favors charge recombination, whereas strong dipole moment favors the charge separation [21,29]. 


\subsection{Donor-Acceptor Separation and Orbital Energy Offset}

Another important parameter that plays a fundamental role in the OPV device performance is the equilibrium separation between donor and acceptor molecules because it controls the electron transfer process from donor to acceptor. The electron transfer process occurs through the proper overlap of electronic wave functions. For the maximum overlap of electronic wave functions, the donor-acceptor distance has to be very short $(\leq 4 \AA)$ [30]. To compute the equilibrium separation of DTBT and C70 in DTBT/C70 complexes, isolated C70 and DTBT planar conformations were initially optimized and C70 molecule was placed through its hexagonal face close to the central hexagonal ring of each of the DTBT conformations. Then the energies of each of the DTBT/C70 complexes were computed at several constrained distances $\left(\mathrm{r}_{\mathrm{D}-\mathrm{A}}\right)$ between BTDT and C70, in the range of $2.7 \AA \leq \mathrm{r}_{\mathrm{D}-\mathrm{A}} \geq 5 \AA$ using dispersion corrected B3LYP.

Figure 5 shows the variation of relative energy of DTBT/C70 complex as a function of $\mathrm{r}_{\mathrm{D}-\mathrm{A}}$ for the DTBT conformation at $\alpha=\beta=180^{\circ}$. Starting from $\mathrm{r}_{\mathrm{D}-\mathrm{A}}=2.7 \AA$, the energy of the complex decreases continuously until $\mathrm{r}_{\mathrm{D}-\mathrm{A}}$ reaches to $\sim 3.2 \AA$. The energy of the complex increases when $\mathrm{r}_{\mathrm{D}-\mathrm{A}}>3.2 \AA$, indicating the equilibrium separation of $3.2 \AA$ between DTBT and C70. This is also true for other complexes of planar DTBT conformations at $\alpha=\beta=0^{\circ}$, and $\alpha=0^{\circ}$ and $\beta=180^{\circ}$. The equilibrium separation of 3.2 $\AA$ also remains unaffected for the complexes when the C70 molecule is placed with its pentagonal face close to the central ring of the DTBT conformations. In a practical OPV device, the backbone conformations are also controlled by intermolecular interactions between different molecules and hence, the equilibrium separation could be slightly different from the one computed in a vacuum as in the present study. Nevertheless, 
equilibrium separation between DTBT and C70 is shorter than that of P3HT/fullerene or PTB7/fullerene ( 3.5 $\AA)$ complexes [21]. This suggests that DTBT-based polymers have more potential to be efficient electron donors for OPV devices as DTBT favors more overlap of electronic wave functions.

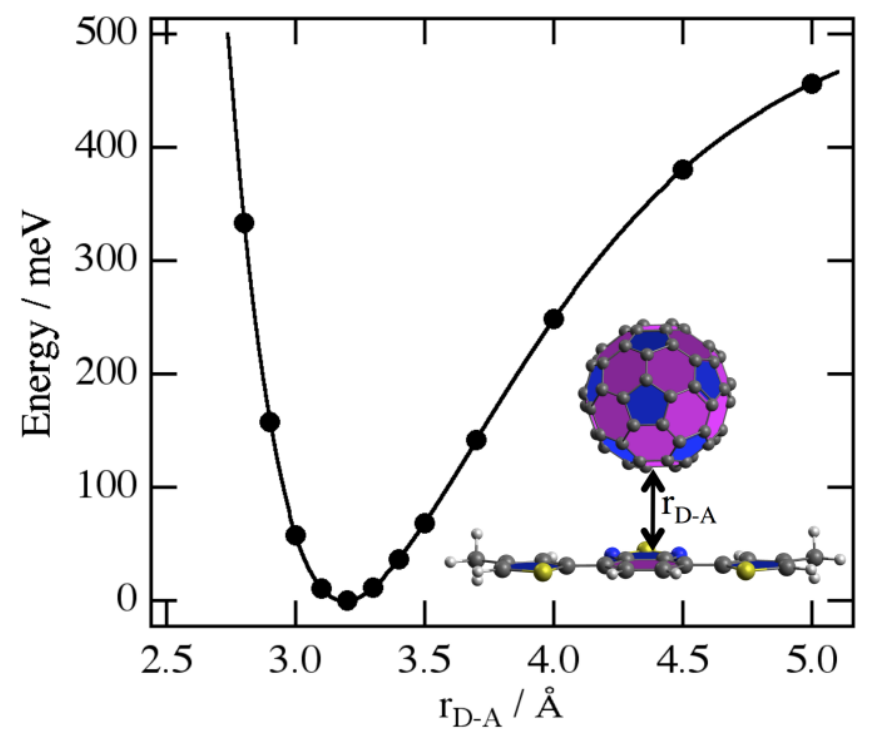

Figure 5. Relative energies of DTBT/C70 complexes as functions of their separation $\left(\mathrm{r}_{\mathrm{D}-\mathrm{A}}\right)$.

The alignment of the frontier molecular orbital energy levels at the donoracceptor interface is also another important parameter for improved performance of OPV devices. Particularly, the energy difference between HOMO levels of DTBT and C70 in the DTBT/C70 complex, called the HOMO offset, is a crucial parameter. Generally, a higher magnitude of HOMO offset suggests an efficient hole transfer, which promotes the charge dissociation at the interface [31]. In contrary, a lower magnitude of HOMO offset implies the charge recombination.

In the present study, HOMO level of DTBT is found to be decreased by $\sim 96 \mathrm{meV}$ and that of the $\mathrm{C} 70$ is found to be increased by $\sim 89 \mathrm{meV}$ in the complex compared to the 
HOMO levels of the isolated DTBT and C70, respectively as a result of the change in dipole moment. This change in HOMO levels in the DTBT/C70 complex is consistent with the reported change in HOMO levels in pentacene/C60 complex [31]. LUMO levels are also found to be affected during the DTBT/C70 complex formation. The LUMO level of DTBT is found to be decreased by $\sim 38 \mathrm{meV}$ and that of $\mathrm{C} 70$ is found to be increased by $\sim 139 \mathrm{meV}$ in the complex compared to the LUMO levels of the isolated DTBT and C70, respectively. The comparison of HOMO offset in C70 complexes of planar DTBT conformations suggests that the complex with DTBT at $\alpha=\beta=180^{\circ}$ conformation favors efficient hole transfer.

\section{CONCLUSIONS}

In conclusion, we have found using first-principle calculations that the trans conformation of DTBT is its lowest-energy conformation in agreement with the experimental report of Osaka et al. The energy of the cis-trans conformation of DTBT that was reported by Stuart et al. as the most stable conformation is about $1.3 \mathrm{kT}$ higher than that of the DTBT trans-conformation. In addition, DTBT in its trans-conformation was found to have lower HOMO-LUMO gap and higher ground-to-excited state dipole difference. Furthermore, DTBT favors shorter equilibrium separation with acceptor molecules compared to that of P3HT or PTB7. These results suggest that DTBT-based polymers have potential to be efficient electron donors for OPV devices. 


\section{Acknowledgements}

We are grateful to the National Science Foundation (Grant No. DMR0847580) for financial support. This work used the extreme science and engineering discovery environment (XSEDE), which is supported by National Science Foundation Grant no. ACI-1053575. The authors thank additional support from the University of Akron. 


\section{References}

[1] Brabec CJ, Sariciftci NS, Hummelen JC. Plastic solar cells. Adv. Funct. Mater. 2001; 11: 15-26.

[2] Sariciftci NS. Plastic photovoltaic devices. Mater. Today 2004; 7: 36-40.

[3] Darling SB, You F. The case for organic photovoltaics. R. Soc. Chem. Adv. 2013; 3: $17633-17648$.

[4] Yu G, Gao J, Hummelen JC, Wudl F, Heeger AJ. Polymer photovoltaic cells: enhanced efficiencies via a network of internal donor-acceptor heterojunctions. Science 1995; 270: 1789- 1791.

[5] Dang MT, Hirsch L, Guillaume W. P3HT:PCBM, best seller in polymer photovoltaic research. Adv. Mater. 2011; 23: 3597-3602.

[6] Thompson BC, Frechet JM. Polymer-Fullerene composite solar cells. Angew. Chem., Int. Ed. 2008; 47: 58-77.

[7] Chen W, Nikiforov MP, Darling SB. Morphology characterization in organic and hybrid solar cells. Energy Environ. Sci. 2012; 5: 8045-8074.

[8] Liang Y, Feng D, Wu Y, Tsai S, Li G, Ray C, Yu L. Highly efficient solar cell polymers developed via fine-tuning of structural and electronic properties. J. Am. Chem. Soc. 2009; 131: 7792-7799.

[9] Stuart AC, Tumbleston JR, Zhou H, Li W, Liu S, Ade H, You W. Fluorine substituents reduce charge recombination and drive structure and morphology development in polymer solar cells. J. Am. Chem. Soc. 2013; 135: 1806-1815.

[10] Osaka I, Shimawaki M, Mori H, Doi I, Miyazaki E, Koganezawa K, Takimiya K. Synthesis, characterization, and transistor and solar cell applications of a 
naphthobisthiadiazole-based semiconducting polymer. J. Am. Chem. Soc. 2012; 134: 3498-3507.

[11] Hohenberg P, Kohn W. Inhomogeneous electron gas. Phys. Rev. 1964; 136: B864B871.

[12] Becke AD. Density-functional thermochemistry. III. The role of exact exchange. J. Chem. Phys. 1993; 98: 5648-5652.

[13] Grimme S. Semiempirical GGA-type density functional constructed with a longrange dispersion correction. J. Comput. Chem. 2006; 27: 1787-1799.

[14] Frisch MJ. et al. Gaussian 09, revision D.01; Gaussian, Inc.: Wallingford, CT, 2013.

[15] Moller C, Plesset MS. Note on an approximation treatment for many-electron systems. Phys. Rev. 1934; 46: 618-622.

[16] Yanai T, Tew D, Handy N. A new hybrid exchange-correlation functional using the coulomb-attenuating method (CAM-B3LYP). Chem. Phys. Lett. 2004; 393: 51-57.

[17] Vydrov OA, Scuseria GE. Assessment of a long range corrected hybrid functional. J. Chem. Phys. 2006; 125: 234109-234118.

[18] Iikura H, Tsuneda T, Yanai T, Hirao K. Long-range correction scheme for generalized-gradient-approximation exchange functionals. J. Chem. Phys. 2001; 115: $3540-3544$.

[19] Bhatta RS, Perry DS, Tsige M. Nanostructures and electronic properties of a highefficiency electron-donating polymer. J. Phys. Chem. A 2013; 117: 12628-12634.

[20] Bhatta RS, Yimer Y, Perry DS, Tsige M. Improved force field for molecular modeling of poly(3-Hexylthiophene). J. Phys. Chem. B 2013; 117: 10035-10045. 
[21] Bhatta RS, Tsige M. Effect of fluorination on electronic properties of polythienothiophene-co-benzodithiophenes and their fullerene complexes. ACS Appl. Mater. Interfaces 2014; 6: 15889-15896.

[22] Ling L, Lagowski JB. DFT study of electronic band structure of alternating triphenylamine-fluorene copolymers. Polymer 2013; 54: 2535-2543.

[23] Li Y, Lagowski JB. Charge carrier mobility in conjugated organic polymers-case studies using multi-step computational approach. Polymer 2011; 52: 4841- 4850.

[24] Bhatta RS, Tsige M. Chain length and torsional dependence of exciton binding energies in P3HT and PTB7 conjugated polymers: A first principles study. Polymer 2014; 55: 2667-2672.

[25] Bhatta RS, Tsige M. Understanding the effect of heteroatoms on structural and electronic properties of conjugated polymers. Polymer 2015; 56: 293-299.

[26] Runge E, Gross EKU. Density-functional theory for time-dependent systems. Phys. Rev. Lett. 1984; 52: 997-1000.

[27] Bhatta RS, Perry D. Correlated backbone torsional potentials in poly(3methylthiophene). Comp. Theor. Chem. 2013; 1008: 90-95.

[28] Risko C, McGehee MD, Bredas J. A Quantum-chemical perspective into low optical-gap polymers for highly-efficient organic solar cells. Chem. Sci. 2011; 2: 1200-1218.

[29] Carsten B, Szarko JM, Son HJ, Wang W, Lu L, He F, Rolczynski BS, Lou SJ, Chen LX, Yu L. Examining the effect of the dipole moment on charge separation in donoracceptor polymers for organic photovoltaic applications. J. Am. Chem. Soc. 2011; 133: 20468-20475. 
[30] Rice MJ, Gartstein YN. Theory of photoinduced charge transfer in a molecularly doped conjugated polymer. Phys. Rev. B 1996; 53: 10764-10770.

[31] Linares M, Beljonne D, Cornil J, Lancaster K, Bredas J, Verlaak S, Mityashin A, Heremans P, Fuchs A, Lennartz C, Ide J, Mereau R, Aurel P, Ducasse L, Castet F. On the interface dipole at the pentacene-fullerene heterojunction: A theoretical study. J. Phys. Chem. C 2010; 114: 3215-3224. 


\section{TOC Graphic}

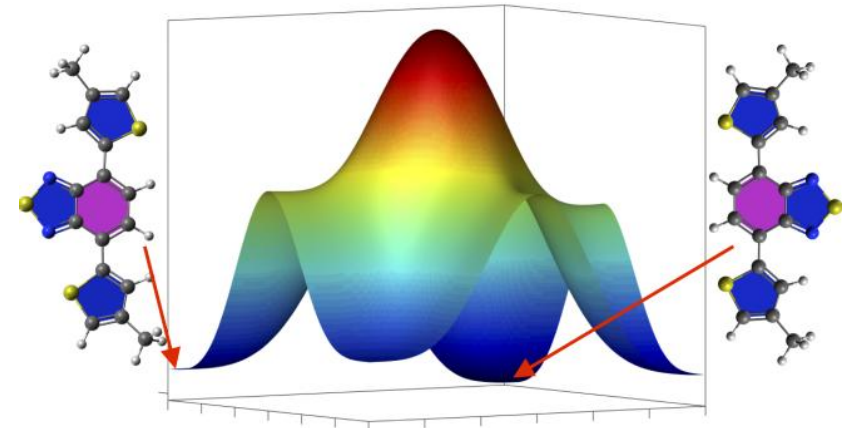

